\title{
Generation of Nondiffracting Electron Bessel Beams
}

\author{
Vincenzo Grillo, ${ }^{1}$ Ebrahim Karimi, ${ }^{2, *}$ Gian Carlo Gazzadi, ${ }^{1}$ Stefano Frabboni, ${ }^{1,3}$ \\ Mark R. Dennis, ${ }^{4}$ and Robert W. Boyd ${ }^{2,5}$ \\ ${ }^{1}$ CNR-Istituto Nanoscienze, Centro S3, Via G Campi 213/a, I-41125 Modena, Italy \\ ${ }^{2}$ Department of Physics, University of Ottawa, 150 Louis Pasteur, Ottawa, Ontario K1N 6N5, Canada \\ ${ }^{3}$ Dipartimento di Fisica Informatica Matemtica (FIM), Università di Modena e Reggio Emilia, \\ via G Campi 213/a, I-41125 Modena, Italy \\ ${ }^{4}$ H. H. Wills Physics Laboratory, University of Bristol, Bristol BS8 1TL, United Kingdom \\ ${ }^{5}$ Institute of Optics, University of Rochester, Rochester, New York 14627, USA \\ (Received 23 October 2013; revised manuscript received 19 December 2013; published 30 January 2014)
}

Almost 30 years ago, Durnin discovered that an optical beam with a transverse intensity profile in the form of a Bessel function of the first order is immune to the effects of diffraction. Unlike most laser beams, which spread upon propagation, the transverse distribution of these Bessel beams remains constant. Electrons also obey a wave equation (the Schrödinger equation), and therefore Bessel beams also exist for electron waves. We generate an electron Bessel beam by diffracting electrons from a nanoscale phase hologram. The hologram imposes a conical phase structure on the electron wave-packet spectrum, thus transforming it into a conical superposition of infinite plane waves, that is, a Bessel beam. We verify experimentally that these beams can propagate for $0.6 \mathrm{~m}$ without measurable spreading and can also reconstruct their intensity distributions after being partially obstructed by an obstacle. Finally, we show by numerical calculations that the performance of an electron microscope can be increased dramatically through use of these beams.

DOI: 10.1103/PhysRevX.4.011013

Subject Areas: Optics, Quantum Physics

\section{INTRODUCTION}

Wave-particle duality lies at the heart of quantum mechanics and serves one of the most important concepts of quantum complementarity, where any particle behaves as a particle or a wave-but, not both simultaneously [1]. A quantum object exhibits its "wavelike" nature during propagation and exhibits its "particlelike" nature when it is detected [1]. The wave-packet concept, then, is introduced to describe the time-space probability distributions, linking both wave and particle natures. Diffraction, the spreading of the wave packet upon propagation, is inherently the soul of the wave nature that is particularly well studied in optics. An optical beam, i.e., a solution to the paraxial wave or Helmholtz equation, expands throughout propagation. Hitherto, two major optical solutions to the wave equation are known, Airy and Bessel beams, which are immune to diffraction in the sense that they do not expand upon propagation $[2,3]$. It is worth noticing that other types of diffraction-free wave packets can be expanded in the Bessel-beam basis [4,5]. An Airy beam preserves its intensity shape during propagation and forms a parabolic

*ekarimi@uottawa.ca

Published by the American Physical Society under the terms of the Creative Commons Attribution 3.0 License. Further distribution of this work must maintain attribution to the author(s) and the published article's title, journal citation, and DOI. curve, similar to a free projectile trajectory, in the transverse-propagation plane. Thus, it is known as an accelerating beam [3,6]. Being an accelerating beam, nonetheless, is in agreement with conservation of linear momentum, since the intensity centroid of an Airy beam remains on a straight line [3]. Airy and Bessel beams are both self-healing, which means that they recover their intensity profiles after being partially obstructed by an obstacle. This remarkable feature exhibits their robustness against any perturbations, such as those introduced by scattering and turbulence [7]. More recently, Voloch-Bloch et al. generated Airy electron beams by implementing a holographic approach in a transmission electron microscope [8]. They transformed an electron wave function into an Airy pattern by implementing cubic phase modulation in the transverse plane. Indeed, they observed both selfbending and self-healing features of this specific wave packet during propagation and obstruction of the beam by a small object, respectively. Apart from being diffraction free, a Bessel beam possesses a couple of significant features, providing remarkable practical applications. It has been so far shown that a Bessel beam of the zeroth order possesses the smallest central-spot diameter compared to other ordinary types of beams [9]. Therefore, it provides a very long uniform localized intensity at the center, applicable in three-dimensional optical trapping $[10,11]$, microscopy [12,13], and laser micromachining [14]. Generating an ideal Bessel beam is not possible since 
it is not power normalizable; a Bessel beam is a coherent superposition of conical plane waves along a closed ring (circle). Nonetheless, quasi-Bessel beams are practical within a good approximate framework. In a very early method, diffraction from a narrow ring aperture was proposed to generate Bessel beams at the aperture far-field plane [15]. The quality of the Bessel beam generated by this approach depends directly on the ring size, whereas confined wave vectors result in a better beam quality at the cost of loss of power of the generated beam. A couple of other different techniques with better efficiency, such as axicon lenses [16], holography [17], and conical diffraction [18], were proposed and verified experimentally to generate quasi-Bessel beams in the optical domain within a certain propagation range.

\section{BESSEL WAVE FUNCTIONS}

Bessel wave functions given by

$$
\psi(\rho, \phi, z ; t)=J_{n}\left(k_{\rho} \rho\right) e^{i n \phi} e^{-i\left(\omega t-k_{z} z\right)}
$$

in the $\rho, \phi, z$ cylindrical coordinates are a well-known solution to the Schrödinger equation of a free electron (or particle), where $J_{n}$ is an $n$ th-order Bessel function of the first kind, $n$ being an integer number, and $k_{\rho}$ and $k_{z}$ are transverse and longitudinal components of the wave vector, respectively. The electron energy defines the corresponding de Broglie wavelength $\left(\lambda_{\mathrm{dB}}\right)$ as well as the strength of the wave vector $(\sqrt{2 m \omega / \hbar})$, i.e., $k^{2}=k_{\rho}^{2}+k_{z}^{2}=2 m \omega / \hbar=$ $\left(2 \pi / \lambda_{\mathrm{dB}}\right)^{2}$, where $m$ and $\hbar$ stand for the electron mass and the reduced Planck constant, respectively. As can be seen in Eq. (1), the probability-density distribution $\mathcal{P}:=\psi^{\dagger} \psi$ of the electrons is independent of $z$ and $t$, i.e., $\mathcal{P}(\rho, \phi, z ; t)=$ $J_{n}\left(k_{\rho} \rho\right)^{2}=\mathcal{P}(\rho, \phi, 0 ; 0)$. Therefore, the probabilitydensity distribution of the electrons in the transverse plane is stationary and independent of plane's location.

For a nonzero $n$ value, electrons possess a nonuniform phase front, forming a helical shape with $n$ dislocations, in which the helicity handedness of the phase front is defined by the sign of $n$ [19]. The current density of electrons circulates azimuthally in the transverse plane and introduces an orbital angular momentum, a quantized "Bohr magneton," of $n \hbar$ per electron. Quantized Bohr magneton associated with electron's orbital angular momentum is in addition is in addition to the already known dipole moment introduced by the electron spin. Unlike the electron spin, as has been shown experimentally, the electron's orbital angular momentum [20,21] can take values much higher than $|\hbar / 2|$, even up to several hundred $|\hbar|$ [22]. Electron's orbital angular momentum provides provides immense practical applications, some of which are discussed in Refs. [23-25]. Thus, electron Bessel beams with a nonzero $n$ value possess $n \hbar$ of angular momentum per electron and have a shape with multiple rings with a null probability density at the origin. More recently, these wave functions were also predicted for a relativistic case as a solution to the
Dirac equation, confirming the electron's $g$ factor of 2 in these solutions [26].

\section{KINOFORM MANUFACTURING}

We implement one of the optical approaches, the holographic method, to generate electron quasi-Bessel beams. Similar to the optics counterpart, a conical phase is imprinted onto the initial electron's wave packet by a nanofabricated kinoform. A kinoform is a pure phase structure with no absorption. It thus alters the electron phase but does not change the probability distribution in the near-field region. Figure 1(a) shows the nanoscale-manufactured kinoform for generating an electron Bessel beam of the zeroth order. An ideal kinoform imprints the following binary phase modulation to the transmitted electrons:

$$
\Phi(\rho, \phi, z)=\Phi_{0} \operatorname{sgn}\left[\cos \left(k_{\rho} \rho+n \phi+\frac{2 \pi}{\Lambda} \rho \cos \phi\right)\right],
$$
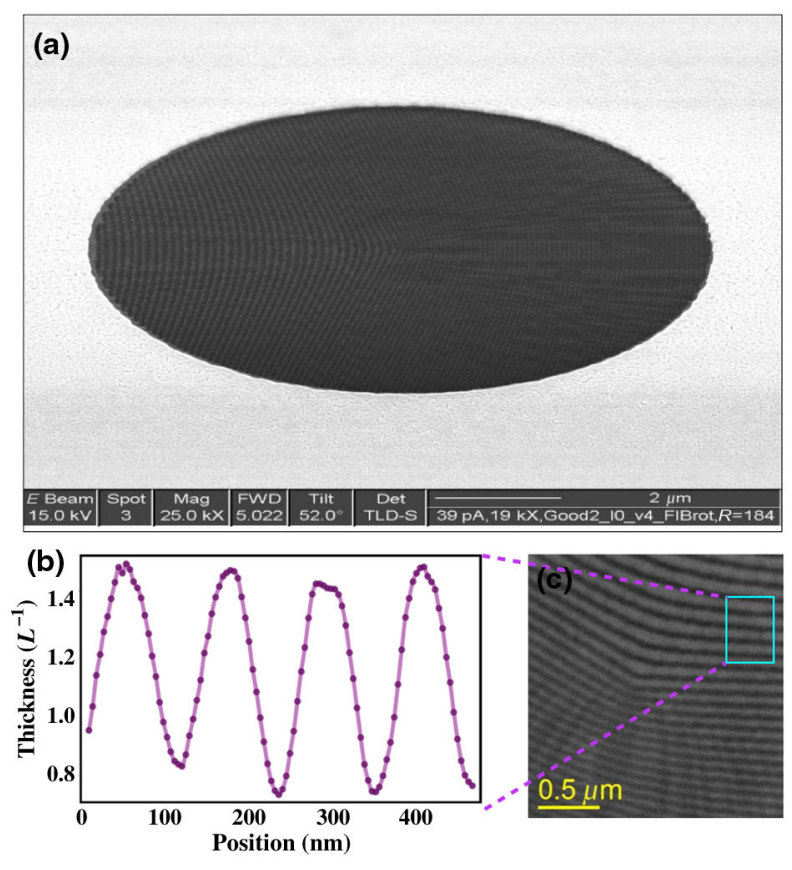

FIG. 1 (color online). (a) Scanning-electron-microscope image (in a tilted direction) of the manufactured kinoform with a zoomin image of the central region shown in (c). The kinoform is made of milled silicon nitride $\left(\mathrm{Si}_{3} \mathrm{~N}_{4}\right)$, which is transparent to the electron beam emitted in the transmission electron microscope. Different depths modify the local atomic potential; thus, electrons see different effective paths (analogous to "optical paths") at grooves. (b) The thickness profile of the silicon nitride calculated by energy-loss mapping of the close-up region in (c). The thickness is expressed in units of $L$, the inelastic electron mean-free path in $\mathrm{Si}_{3} \mathrm{~N}_{4}$. The estimated value of $L$ is about $130 \mathrm{~nm}$. The profile is largely nonrectangular and closer to a sinusoidal form. This depth results in an effective phase change of $1.6 \pi$ for electrons with a de Broglie wavelength of $\lambda_{\mathrm{dB}}=2.5 \mathrm{pm}$. 
where $\Phi_{0}$ and $\Lambda$, define the modulation depth and the grating period, respectively, and sgn is the sign function. The designed kinoform tailors a conical phase front onto the electron's wave packet at the first order of diffraction. Thus, the wave packet forms a Bessel beam in the far-field plane of the kinoform. This binary kinoform diffracts electrons into all odd diffraction orders, whereas the phase modulation of $\Phi_{0}$ adjusts the diffraction power. Indeed, we use a dual-beam instrument (FEI Strata DB235M), combining a Ga-ion focused-ion beam (FIB) and a scanning electron microscope to pattern the kinoforms [Eq. (2)] by FIB milling on silicon-nitride membranes coated with a gold film. The 200-nm-thick membrane is coated with a 120-nm-thick gold film in order to stop forward electron transmission except from the patterned areas. The procedure for pattern nanofabrication starts with a computergenerated bitmap image of the hologram that is converted into a FIB pattern file containing (i) the pixel coordinates where the FIB is switched on, (ii) the beam-dwell time on each pixel, and (iii) the repetition number of the whole coordinate set, adjusted in such a way as to obtain the desired milling depth. The second step is to adjust the FIB magnification according to the desired dimension of the hologram. We select a 50 -nm width and $100-\mathrm{nm}$ periodicity for the stripes composing the hologram, resulting in a full hologram size of $10 \mu \mathrm{m} \times 10 \mu \mathrm{m}$. Finally, the kinoforms are patterned in two stages: First, the gold layer is uniformly removed by a FIB from a $10-\mu \mathrm{m}$ round area; then, the hologram pattern is superimposed to the uncovered region and milled into the silicon nitride. For an ideal binary kinoform, the power of diffracted electrons at the first order of diffraction can reach up to approximately $40 \%$, which is about 4 times higher than that generated by the amplitude holograms reported in prior works. However, the effectively generated pattern in the FIB turns into an approximatively sinusoidal form, and thus the efficiency of the generated beam is approximately $17 \%$ smaller than an ideal binary profile (40\%) with the same depth. Figure 1(b) shows the thickness profile of the kinoform, calculated by energy-loss mapping, for a closed-up region indicated in Fig. 1(c).

\section{EXPERIMENTAL RESULTS}

In our experiment, a relatively coherent monoenergetic electron beam is produced and imaged using a JEOL $2200 \mathrm{Fx}$ transmission electron microscope operated at a central energy of $\mathcal{E}=200 \mathrm{keV}\left(\delta \mathcal{E} / \mathcal{E}<5 \times 10^{-6}\right)$ and equipped with a Schottky-field emitter. The associated de Broglie wavelength, including relativistic correction, is about $\lambda_{\mathrm{dB}}=2.5 \mathrm{pm}$. The experiments are performed by introducing the kinoform into the sample position and using the "low-magnification mode" (corresponding to the Lorenz mode), where the main objective lens is turned off. A systematic variation of the other lenses (in particular, the objective minilens) permits us to move from an
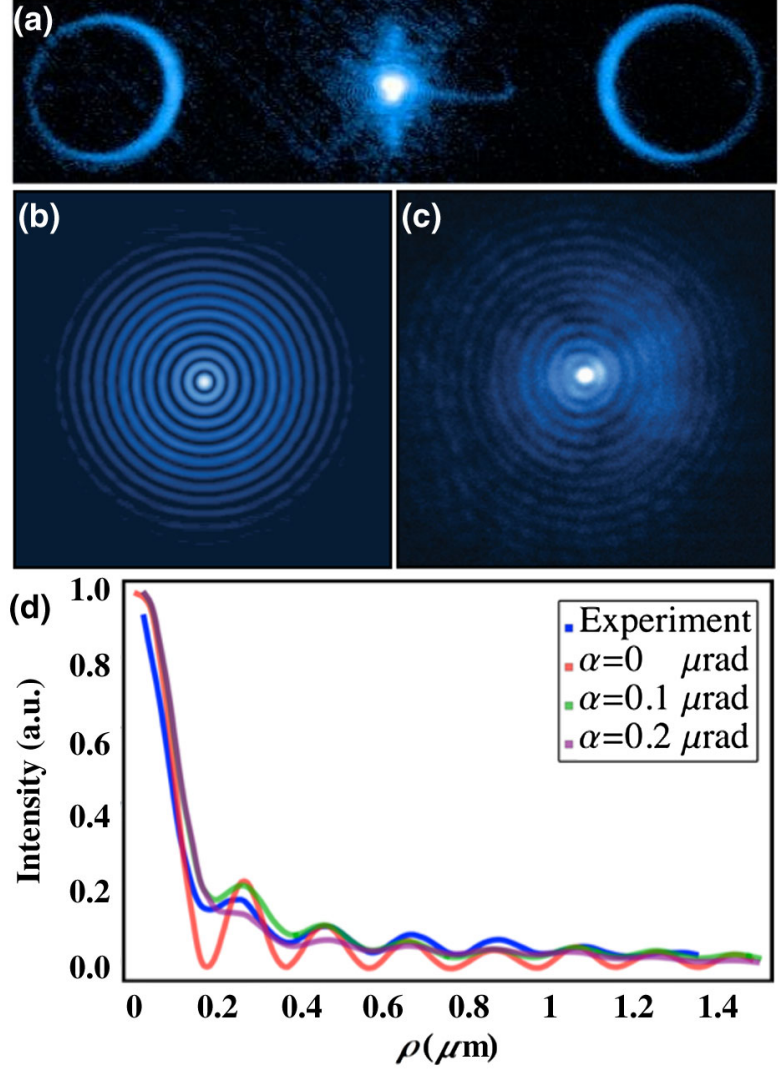

FIG. 2 (color online). (a) Far-field distribution of the diffracted electrons by the kinoform shown in Fig. 1(a). Only the zeroth and first orders are shown. In the first order of diffraction, the Bessel beam forms a ring in the far field. (b) Simulated transverse distribution of the electron Bessel beam of the zeroth kind at the first order of diffraction. (c) Measured transverse distribution of the electron Bessel beam of the zeroth order generated by the kinoform in Fig. 1(a). The number of symmetric multirings present defines the imprinted conical phase front to the electron wave packet. (d) Radial distribution of the detected electrons (solid blue curve) for the electron Bessel wave function. The plot legend shows the simulated Bessel beams with different convergences $\alpha$ on the kinoform plane.

image plane to the Fresnel and Fraunhofer diffraction regions. This special unconventional working mode permits us to obtain an illumination-convergence angle below $1 \mu \mathrm{rad}$; such a convergence angle is necessary since the typical diffraction angles in the hologram are of some tens of $\mu \mathrm{rad}$. The beam size, magnification, and defoci are accurately recalibrated for this unconventional working mode using a reference hologram and comparing with simulations of the Fresnel patterns. In all cases, the kinoform is illuminated by an approximate electron plane wave with a convergence angle below $0.4 \mu \mathrm{rad}$. The distribution of electrons at the first order of diffraction after passing through the hologram is then recorded between the image plane and the back focal plane of magnetic lenses using the above approach. Numerically simulated and experimentally observed intensity distributions of electrons at the focal plane of the kinoform are shown 

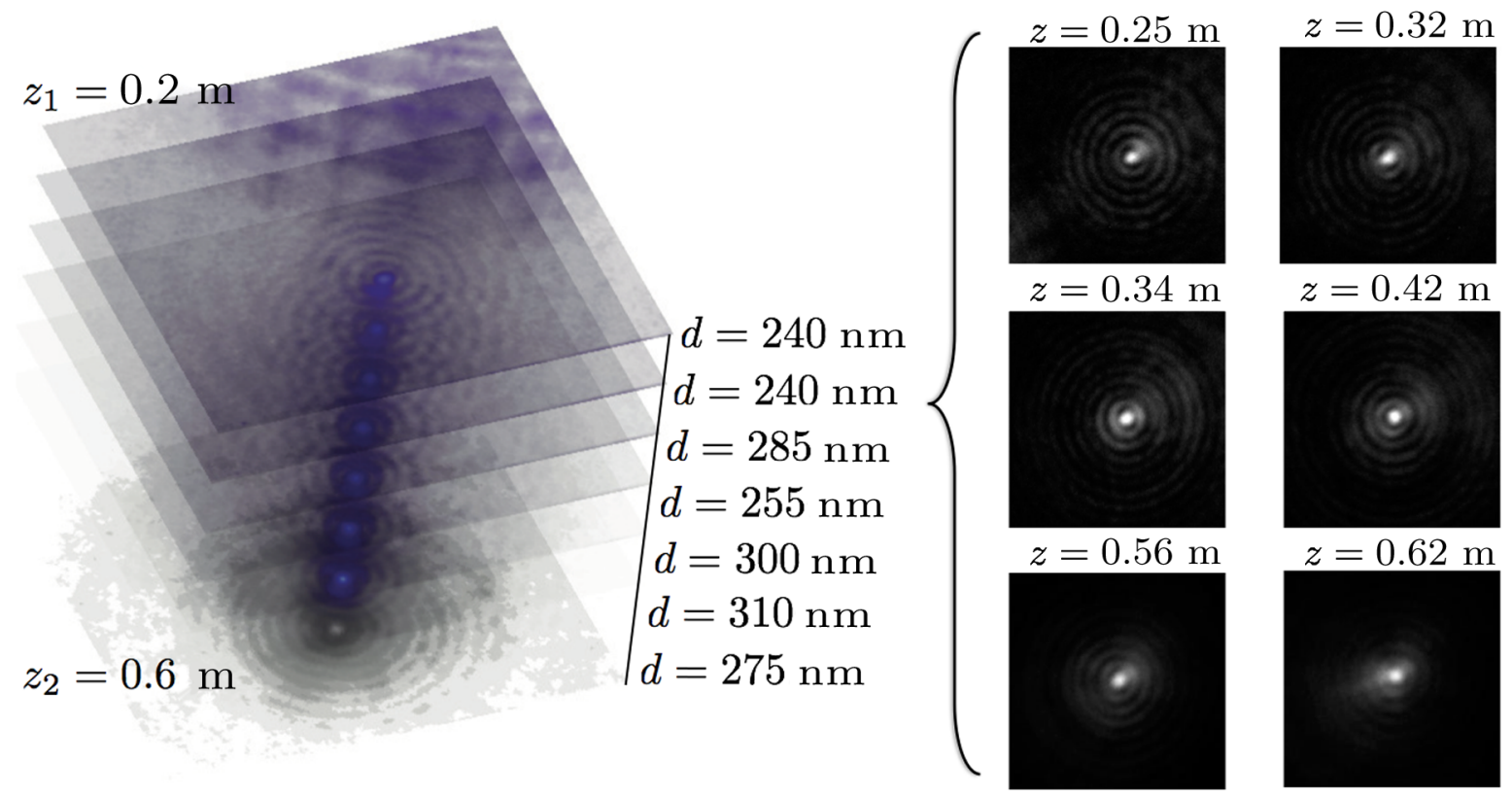

FIG. 3 (color online). Observed image of the probability-density distribution of electrons for a Bessel beam of the zeroth order in different propagation planes. Note that the beam diameter remains mainly constant as a function of propagation distance. The small variation in the radius (the distance from the central to the first ring) occurs because we are using an approximate Bessel beam. This variation has already been predicted and observed in the optical domain [9]. The right inset shows the raw images of electron distributions upon propagation at different plans.

in Figs. 2(b) and 2(c), respectively. Adjusting the transverse momentum, $k_{\rho}$ in Eq. (2) alters the hologram-tilting anglesthus changing the intensity pattern of the generated beam. Multiple rings seen in Fig. 2(c) confirm the generation of an electron Bessel beam. The interference of conical plane waves gives rise to cylindrically symmetric fringes. The radius of the $k$ th null ring is given by $\rho_{0, k}=j_{0, k} / k_{\rho}$, where $j_{0, k}$ is the $k$ th positive zero of the Bessel function of the zeroth order [27]. The radius of the central spot is thus $\rho_{0,1}=j_{0,1} / k_{\rho}$, which is $\rho_{0,1}=2.04 / k_{\rho}$. The probability distribution for an ideal Bessel beam at each ring is identical. Thus, as is expected, it requires infinite power.

\section{A. Nondiffracting feature}

In order to verify the existence of the diffraction-free feature, we record the probability distribution of the diffracted electrons at the first order of diffraction for different planes near the back focal plane. For a kinoform with a conical alteration, analogous to an axicon, the diffractionfree range is given by $\mathcal{Z}=k w_{0} / k_{\rho}$, where $w_{0}$ stands for the kinoform aperture [28]. A theoretical estimation of the diffraction-free range of the manufactured hologram is $\mathcal{Z} \simeq 0.75 \mathrm{~m}$. Figure 3 shows a three-dimensional image of the probability distribution of electrons possessing the Bessel wave function at various propagation distances within the Fresnel regime at locations between the image and the focal plane of the kinoform. For a propagation distance range of $0.2-0.6 \mathrm{~m}$, the probability distribution of the electron Bessel beam does not change, showing that these beams are resistant to the effects of diffraction. The Rayleigh range with a conventional electron beam with the same spot size is approximately $0.16 \mathrm{~m}$. The increased range of the Bessel beam matches quite well with our simulated prediction, i.e., $0.75 \mathrm{~m}$.

\section{B. Higher-order Bessel beams}

We also manufacture pure phase holograms for generating higher-order electron Bessel beams by setting different
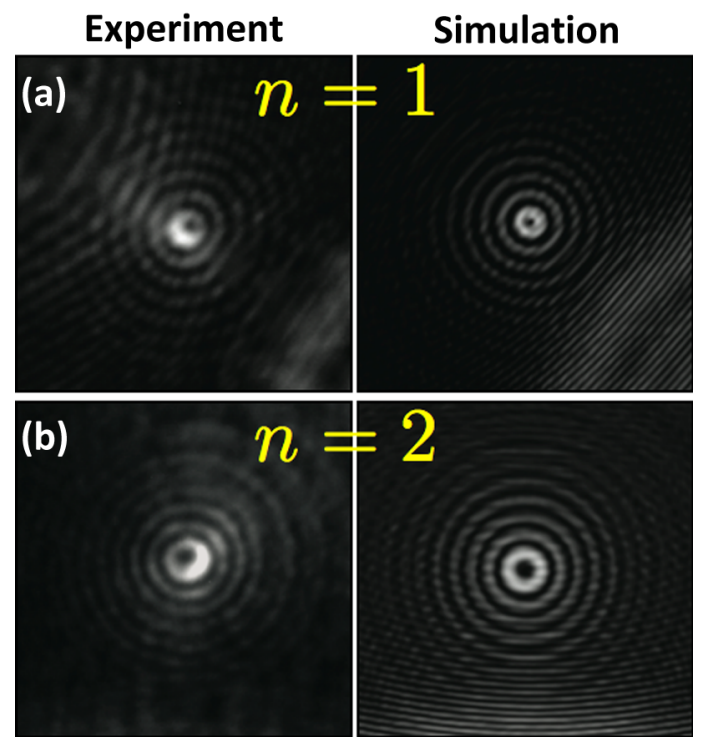

FIG. 4 (color online). Experimentally observed (left panels) and simulated (right panels) images of the higher-order electron Bessel beams with topological charges of $n=1$ and $n=2$. 
(a)

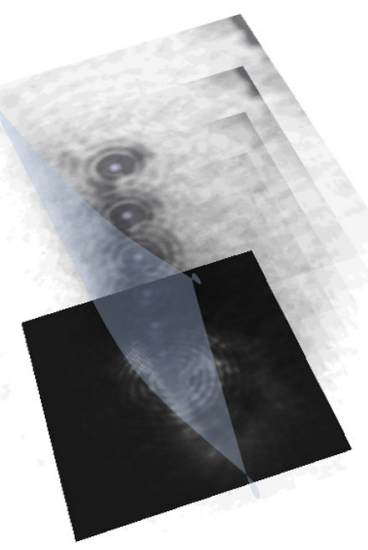

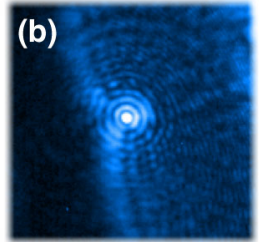

(c)

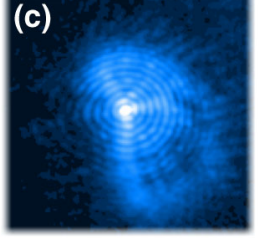

FIG. 5 (color online). (a) Intensity distribution of the electron Bessel beam of the zeroth order upon propagation after being obstructed by an asymmetrical obstacle. (b),(c) The distribution of the electrons after the obstacle and at the plane of $0.1 \mathrm{~m}$. As seen in (c), the beam recovers its "Bessel-like" intensity distribution, revealing the self-healing feature.

nonzero topological charges of $n$ in Eq. (2). These beams possess vortices at the origin, and each electron carries a well-defined value of orbital angular momentum of $n \hbar$. Figures 4(a) and 4(b) show simulated and experimentally observed higher-order Bessel beams generated by kinoforms of Eq. (2) with $n=1$ and $n=2$, respectively.

\section{Self-healing}

As the final test, we examine the self-healing property of the generated electron Bessel beam. We put an aperture at a plane close to the image plane of the kinoform. The aperture obstructs a portion of the beam (about 30\%) and is arranged to be asymmetric with respect to the kinoform center. Then, we record distribution patterns of the electrons during free-space propagation. The intensity patterns of the electron Bessel beam of the zeroth order at various planes after being partially obstructed by an asymmetric aperture are recorded upon propagation. Figure 5 shows the experimental observation of the self-healing of the Bessel beam of the zeroth order. As can be seen, the beam recovers its pattern upon propagation. As is discussed, the electron Bessel beams are a coherent superposition of conical plane waves along a closed ring (circle); thus, obstructing a portion of the beam does not affect the beam-intensity distribution at the far-field region.

\section{APPLICATIONS}

The electron Bessel beam has unique features, such as being diffraction free and self-healing, in addition to possessing a minimum central-spot size. Thus, analogous to light microscopy, it can be used in electron microscopy. Bessel beam of the zeroth order is particularly is particularly

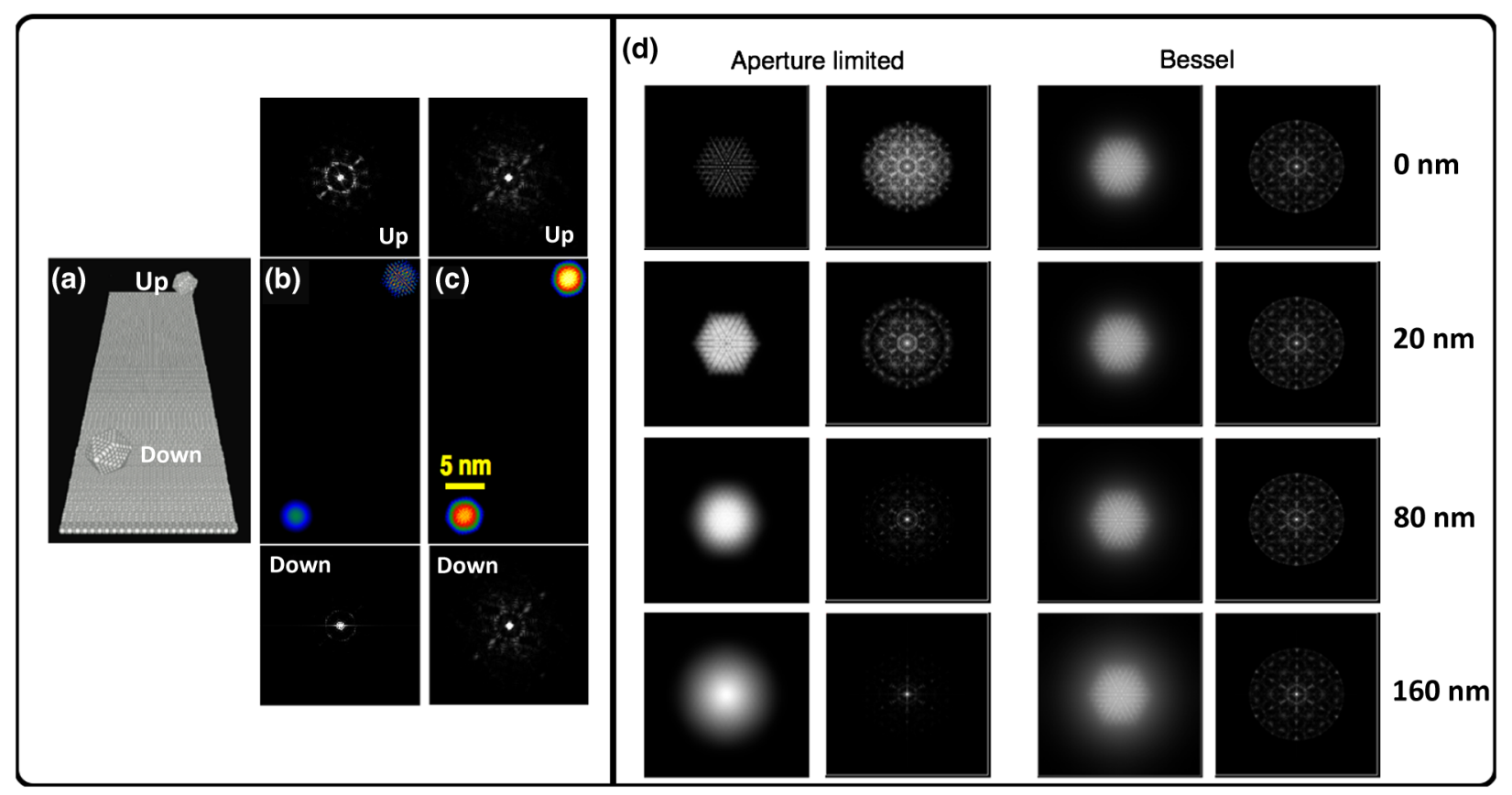

FIG. 6 (color online). Simulated HAADF-STEM image for two particles lying on a $60^{\circ}$-inclined graphene sheet. (a) The two spheres are displaced in depth by $17 \mathrm{~nm}$. Notice that graphene is not visible in the simulation because of its low contrast. (b) The simulated image of the particles with a conventional STEM probe with $\alpha=15 \mathrm{mrad}$. (c) The simulated image of the particles with an electron Bessel probe in a HAADF-STEM. The upper and lower insets in (b) and (c) are Fourier transforms of the images (diffractograms) of the upper and lower particles, respectively. (d) Numerically simulated images (left column) and diffractograms (right column) of a particle located at different planes of a focused aperture-limited probe and a zeroth-order Bessel-beam probe. As is seen, the atomic resolution of the particle is clearly visible for a Bessel-beam probe at different positions upon propagation. A conventional STEM probe with convergence of $\alpha=15 \mathrm{mrad}$ is assumed. 
useful in electron tomography of materials, where an image at different planes of different depths is needed [29]. Indeed, the size and intensity distribution of the electron Bessel beam for different planes at different depths are identical and do not vary by propagation. Furthermore, the probe beam recovers its distribution after being perturbed by obstacles inside the examined sample (recall self-healing). The self-healing range is proportional to obstacle size. Therefore, larger particles acquire a longer reconstruction range. However, we simulate a high-angle annular dark-field scanningtransmission-electron-microscopy (HAADF-STEM) [30] image for two particles lying on a $60^{\circ}$-inclined graphene sheet, shown in Fig. 6(a) for two different electron probebeam shapes: (i) a conventional diffraction-limited probe and (ii) a Bessel beam of the zeroth order. Figures 6(b) and 6(c) show the simulated images in a HAADF-STEM with conventional diffraction-limited and Bessel probes, respectively. The Bessel beam provides a strong convergence up to $20 \mathrm{mrad}$ because it is resistant to the effects of spherical aberration. As seen in Figs. 6(b) and 6(c), the simulation clearly highlights that in the case of a Bessel beam, the particles remain in focus. The above analysis implies that tomography could be performed without the need to adjust the focus at any tilt.

\section{CONCLUSION}

In conclusion, we have performed a holographic approach to generate electron Bessel beams in a middle energy range of transmission electron microscopy. A novel nanofabricated phase hologram for electron beams is presented and implemented for the first time and is used to generate such a beam with a high diffraction efficiency. Far- and near-field probability distributions of generated electrons ensure generation of Bessel beams since they form a narrow ring and cylindrically symmetric multirings, respectively. Also, one of the most exciting features of this beam, i.e., being diffraction free, was observed in a certain region of propagation. Finally, we simulated the importance of a Bessel beam of the zeroth order in image tomography by simulating an image of two out-of-plane icosahedral nanoparticles.

\section{ACKNOWLEDGMENTS}

E. K. and R.W.B. acknowledge the support of the Canada Excellence Research Chairs (CERC) Program.

[1] J. A. Wheeler and W. H. Zurek, Quantum Theory and Measurement (Princeton University, Princeton, NJ, 1983).

[2] J. Durnin, Exact Solutions for Nondiffracting Beams. I. The Scalar Theory, J. Opt. Soc. Am. A 4, 651 (1987).

[3] M. V. Berry and N. L. Balazs, Nonspreading Wave Packets, Am. J. Phys. 47, 264 (1979).

[4] J. A. Stratton, Electromagnetic Theory (John Wiley \& Sons, Hoboken, NJ, 2007).
[5] J. C. Gutiérrez-Vega, M. D. Iturbe-Castillo, and S. Ch'avez-Cerda, Alternative Formulation for Invariant Optical Fields: Mathieu Beams, Opt. Lett. 25, 1493 (2000).

[6] G. A. Siviloglou, J. Broky, A. Dogariu, and D. N. Christodoulides, Observation of Accelerating Airy Beams, Phys. Rev. Lett. 99, 213901 (2007).

[7] J. Baumgartl, M. Mazilu, and K. Dholakia, Optically Mediated Particle Clearing Using Airy Wavepackets, Nat. Photonics 2, 675 (2008).

[8] N. Voloch-Bloch, Y. Lereah, Y. Lilach, A. Gover, and A. Arie, Generation of Electron Airy Beams, Nature (London) 494, 331 (2013).

[9] D. McGloin and K. Dholakia, Bessel Beams: Diffraction in a New Light, Contemp. Phys. 46, 15 (2005).

[10] V. Garcés-Chávez, D. McGloin, M. J. Padgett, W. Dultz, H. Schmitzer, and K. Dholakia, Observation of the Transfer of the Local Angular Momentum Density of a Multiringed Light Beam to an Optically Trapped Particle, Phys. Rev. Lett. 91, 093602 (2003).

[11] V. Garcés-Chávez, D. McGloin, H. Melville, W. Sibbett, and K. Dholakia, Simultaneous Micromanipulation in Multiple Planes Using a Self-Reconstructing Light Beam, Nature (London) 419, 145 (2002).

[12] T. A. Planchon, L. Gao, D. E. Milkie, M. W. Davidson, J. A. Galbraith, C. G. Galbraith, and E. Betzig, Rapid Three-Dimensional Isotropic Imaging of Living Cells Using Bessel Beam Plane Illumination, Nat. Methods 8, 417 (2011).

[13] F. O. Fahrbach, Ph. Simon, and A. Rohrbach, Microscopy with Self-Reconstructing Beams, Nat. Photonics 4, 780 (2010).

[14] M. K. Bhuyan, F. Courvoisier, P.-A. Lacourt, M. Jacquot, L. Furfaro, M. J. Withford, and J. M. Dudley, High Aspect Ratio Taper-Free Microchannel Fabrication Using Femtosecond Bessel Beams, Opt. Express 18, 566 (2010).

[15] J. Durnin, J. J. Miceli, and J. H. Eberly, Diffraction-Free Beams, Phys. Rev. Lett. 58, 1499 (1987).

[16] J. H. McLead, Axicon: A New Type of Optical Element, J. Opt. Soc. Am. 44, 592 (1954).

[17] A. Vasara, J. Turunen, and A. T. Friberg, Realization of General Nondiffracting Beams with Computer-Generated Holograms, J. Opt. Soc. Am. A 6, 1748 (1989).

[18] M. V. Berry, M. R. Jeffrey, and J. G. Lunney, Conical Diffraction: Observations and Theory, Proc. R. Soc. A 462, 1629 (2006).

[19] K. Y. Bliokh, Y. P. Bliokh, S. Savel'ev, and F. Nori, Semiclassical Dynamics of Electron Wave Packet States with Phase Vortices, Phys. Rev. Lett. 99, 190404 (2007).

[20] M. Uchida and A. Tonomura, Generation of Electron Beams Carrying Orbital Angular Momentum, Nature (London) 464, 737 (2010).

[21] J. Verbeeck, H. Tian, and P. Schattschneider, Production and Application of Electron Vortex Beams, Nature (London) 467, 301 (2010).

[22] B. J. McMorran, A. Agrawal, I. M. Anderson, A. A. Herzing, H. J. Lezec, J. J. McClelland, and J. Unguris, Electron Vortex Beams with High Quanta of Orbital Angular Momentum, Science 331, 192 (2011).

[23] J. Verbeeck, P. Schattschneider, S. Lazar, M. Stöger-Pollach, S. Löffler, A. Steiger-Thirsfeld, and G. Van Tendeloo, 
Atomic Scale Electron Vortices for Nanoresearch, Appl. Phys. Lett. 99, 203109 (2011).

[24] E. Karimi, L. Marrucci, V. Grillo, and E. Santamato, Spinto-Orbital Angular Momentum Conversion and SpinPolarization Filtering in Electron Beams, Phys. Rev. Lett. 108, 044801 (2012).

[25] K. Y. Bliokh, P. Schattschneider, J. Verbeeck, and F. Nori, Electron Vortex Beams in a Magnetic Field: A New Twist on Landau Levels and Aharonov-Bohm States, Phys. Rev. X 2 , 041011 (2012).

[26] K. Y. Bliokh, M. R. Dennis, and F. Nori, Relativistic Electron Vortex Beams: Angular Momentum and Spin-Orbit Interaction, Phys. Rev. Lett. 107, 174802 (2011).
[27] M. Abramowitz and I. A. Stegun, Handbook of Mathematical Functions (National Bureau of Standards, Washington, DC, 1972).

[28] G. Indebetouw, Nondiffracting Optical-Fields-Some Remarks on Their Analysis and Synthesis, J. Opt. Soc. Am. A 6, 150 (1989).

[29] P. A. Midgley and R. E. Dunin-Borkowski, Electron Tomography and Holography in Materials Science, Nat. Mater. 8, 271 (2009).

[30] V. Grillo and E. Rotunno, STEM-CELL: A Software Tool for Electron Microscopy: Part I-Simulations, Ultramicroscopy 125, 97 (2013). 